\title{
Boas práticas agropecuárias na produção leiteira: diagnóstico e ajuste de não conformidades
}

\author{
[Good agricultural practices for milk production: diagnosis and \\ non-compliances adjustment] \\ R.M. Dereti ${ }^{1}$, E.B. Gonçalves ${ }^{2}$, M.B. Zanela ${ }^{3}$, \\ J. Schafhauser Júnior ${ }^{3}$, M.B. Alvarenga ${ }^{3}$ \\ ${ }^{1}$ Instituição Embrapa Gado de Leite/NATT-Sul - Juiz de Fora, MG \\ ${ }^{2}$ Instituição Embrapa Agroindustria de Alimentos - Rio de Janeiro, RJ \\ ${ }^{3}$ Instituição Embrapa Clima Temperado - Pelotas, RS
}

\section{RESUMO}

Objetivou-se com este estudo desenvolver e validar uma ferramenta semiológica para diagnóstico do nível de adoção e conformidade das boas práticas agropecuárias em fazendas de produção de leite, segundo requisitos preconizados pela Food and Agriculture Organization of the United Nations (FAO) e pela International Dairy Federation (IDF). A ferramenta foi testada em 62 fazendas de produção de leite, em seis diferentes regiões do estado do Rio Grande do Sul, como parte das ações do Projeto Protambo - "Transferência de tecnologias para o desenvolvimento da atividade leiteira no RS com base nas boas práticas agropecuárias" - da Empresa Brasileira de Pesquisa Agropecuária, Embrapa, entre janeiro de 2014 e março de 2017. A validação da ferramenta não apontou divergências estatisticamente significativas entre avaliadores, satisfazendo o parâmetro de exatidão para métodos de medições exigidos pela ISO/IEC 17025. Foi evidenciado um espaço de variação que mostrou desenvolvimento de métrica, em que se obteve consistência (coerência) de medição. $\mathrm{O}$ teste tStudent aproximado para a comparação de médias de não conformidades nas BPA mostrou melhora significativa no grupo tratamento (diagnóstico seguido de plano de ajuste) quando comparado ao controle (diagnóstico sem plano de ajuste subsequente). Essa nova abordagem semiológica contribui para a adoção das BPA em fazendas leiteiras, para a melhoria da qualidade do leite e da segurança na cadeia produtiva de lácteos.

Palavras-chave: boas práticas agropecuárias, produção de leite, sistemas de produção, qualidade do leite, método de diagnóstico

\begin{abstract}
This study reports the development and validation of a novel diagnostic tool, based on the FAO and IDF "Guide to Good Dairy Farming Practice." Sixty-two dairy farms over six different regions within the State of Rio Grande do Sul were selected, evaluated and ranked, from January 2014 to March 2017; as part of the PROTAMBO- Dairying Technology Transfer Project (EMBRAPA). Results indicated that the proposed diagnostic tool was significantly consistent among different field evaluators, meeting trueness validation parameter for ISO/IEC 17025 validation requirement. Binomial distribution of probabilities of positive changes showed significant kind of metric evolution for the treatment group when compared to the control, in addition to significant consistency. Approximated t-Student test for comparison of the means of GAP noncompliances demonstrated significant improvements for the treatment group relative to the control. This novel approach could assist in overcoming existing and emerging GAP challenges to maximize dairy quality.
\end{abstract}

Keywords: Dairy farming, milk quality, diagnosis tool, Good Agricultural Practices, GAP, production system

\section{INTRODUÇÃO}

O grande desafio para melhoria da qualidade do leite não é limitado exclusivamente pelo conhecimento existente em si, mas também pela

Recebido em 24 de novembro de 2017

Aceito em 15 de abril de 2019

E-mail: rogerio.dereti@embrapa.br capacidade de transformar o conhecimento em prática (More, 2009). O leite é produto de um sistema de produção pecuária, obtido manual ou mecanicamente, que requer práticas de higiene, resfriamento e armazenamento, as quais se aproximam mais de rotinas de processos de fabricação do que de práticas agropecuárias em 
geral. As boas práticas, entretanto, são um conjunto articulado de ações que, após realizadas, acarretam ganhos de produção, produtividade, qualidade, segurança do alimento e sustentabilidade para as fazendas e para toda a cadeia leiteira. Não se trata, apenas, de reduzir a carga microbiana, a ocorrência de leite instável ao teste de acidez, de contaminantes químicos e perigos biológicos, entre outros aspectos que afetam negativamente a qualidade do leite, mas de promover mudanças no ambiente físico das fazendas e transformações de cunho social entre os diversos segmentos do setor lácteo.

Mudanças necessárias à transformação dos padrões de qualidade e segurança na produção de leite requerem um método abrangente e clareza quanto ao que precisa ser feito. $\mathrm{O}$ estabelecimento do nível de aprofundamento necessário em cada aspecto do processo não deve ser apenas conceitual, mas deve incluir a qualificação e especificidade dos indicadores, desafios esses a serem enfrentados. Além dos problemas previamente descritos, somam-se problemas correlatos, como o nível de letramento dos produtores, o preço do leite e as políticas de remuneração por qualidade, entre outros. Há que se reconhecer a dificuldade de se conciliar os requisitos básicos de qualidade e segurança do alimento no contexto da produção leiteira em escala comercial (Brito, 2004; Vallin et al., 2009; Young et al., 2010; Mororó, 2011; Dufour, 2012).

O sucesso dos programas de boas práticas agropecuárias (BPA) esbarra na percepção, por parte dos produtores, de que as boas práticas exercem baixo - ou possivelmente nenhum impacto no resultado final do sistema de produção e na qualidade dos produtos obtidos, e, assim, esses não as reconhecem ao ponto de incorporá-las devidamente (Scalco e Souza, 2006; Vallin et al., 2009; Young et al., 2010). Sob a perspectiva legalista, os regulamentos, as normativas e as recomendações técnicas, tais como as Instruções Normativas e Regulamentos nacionais (IN 51, IN 62, RIISPOA, IN 76, IN 77) e internacionais (Codex Alimentarius, documentos diversos da FAO/IDF, OIE), definem quais são as "boas práticas". Sob a premissa de que regulamentos deveriam ser cumpridos, bastaria treinar as pessoas para a adoção das boas práticas e prover recursos materiais para a melhoria do processo. No entanto, os programas para adoção das boas práticas, em geral, adotam estratégias verticais, que desconsideram a técnica, o conhecimento tácito e a percepção dos produtores acerca de suas ações cotidianas e sobre as práticas a serem adotadas. As BPA são tratadas nesses contextos como metas a serem atingidas.

O Guia de Boas Práticas na Pecuária de Leite da Food and Agriculture Organization of the United Nations (FAO) e da International Dairy Federation (IDF) enumera diretrizes relacionadas à segurança do consumidor e à gestão econômica, social e ambiental de propriedades leiteiras, agrupadas em seis áreas correlatas às boas práticas agropecuárias (BPA) na produção de leite. Ademais, tal documento contém vários elementos específicos que contribuem para as boas práticas na pecuária de leite. Entretanto, o Guia FAO-IDF não traz um instrumento que permita diagnosticar e estabelecer prioridades para orientar planos de adoção ou de ajuste das práticas adotadas e, sobretudo, para a solução das não conformidades pelos produtores. Os programas brasileiros de aplicação mais extensiva também não o fazem (Costa et al., 2018) ou o fazem com abordagem distinta da adotada neste estudo.

O objetivo desta pesquisa foi desenvolver e validar um instrumento (ferramenta Protambo) para o conhecimento da realidade das práticas adotadas nas unidades de produção de leite, que permitisse o estabelecimento de prioridades para elaboração de planos de ajuste de não conformidades, de acordo com o Guia de Boas Práticas na Pecuária de Leite (Guia, 2013).

\section{MATERIAL E MÉTODOS}

A pesquisa foi realizada entre janeiro/2014 e abril/2017, em 62 unidades de produção de leite (UPL) participantes do projeto Protambo "Transferência de tecnologias para o desenvolvimento da atividade leiteira no RS com base nas boas práticas agropecuárias", distribuídas em seis regiões do estado do Rio Grande do Sul: Sudeste, Sul, Noroeste, Central, Serra e Missões. Foi financiada pelo Macroprograma 4, carteira de projetos de Transferência de Tecnologia da Embrapa. Instituições de distintas naturezas jurídicas, públicas e privadas, tais como cooperativas, associações de laticínios e a Empresa de 
Assistência Técnica e Extensão Rural do Estado do Rio Grande do Sul, Emater RS, empresa pública, foram parceiras da Embrapa no projeto Protambo. Essas instituições disponibilizaram um técnico para ser treinado na aplicação do instrumento de diagnóstico (ferramenta Protambo) em desenvolvimento, bem como na formulação e no acompanhamento de planos de ajuste de não conformidades durante os três anos de vigência do projeto. Os técnicos assistentes das UPL tinham diversas formações: técnicos agrícolas e agropecuários, além de veterinários, agrônomos e zootecnistas.

As articulações entre a Embrapa e as instituições parceiras iniciaram-se antes da aprovação do projeto, em 2013, e o início das atividades ocorreu imediatamente após a aprovação. A primeira atividade conjunta foi uma reunião entre todos os envolvidos, na qual foram apresentados os métodos a serem utilizados, os critérios de seleção de propriedades e o cronograma previsto. Nessa reunião o protótipo da ferramenta Protambo de diagnóstico de boas práticas agropecuárias foi apresentado aos técnicos, para familiarização antes do treinamento de campo. As instituições parceiras foram orientadas quanto à seleção das propriedades, segundo os seguintes critérios de elegibilidade: a disposição dos produtores a participar de um programa de boas práticas e de adotar as práticas/mudanças pactuadas com os técnicos; a garantia de acesso às fazendas sempre que necessário; a disposição quanto à cessão dos dados obtidos; e não estar participando de outro programa de boas práticas concomitantemente. Não houve interferência direta da Embrapa na seleção das UPL, uma vez que o projeto buscou atuar com a maior flexibilidade possível quanto aos tipos de sistemas de produção e aos níveis de intensificação tecnológica das UPL, atendendo distintas necessidades das instituições parceiras e de seus associados. Essas premissas delineiam o ambiente adequado para o desenvolvimento de uma metodologia de aplicação ampla e flexível, adaptável à grande diversidade de sistemas de produção de leite na região Sul e no Brasil.

A etapa seguinte foi a seleção e o convite aos produtores, a qual foi realizada pelas instituições parceiras. No momento da inclusão de cada UPL, foi aplicado um questionário para levantar informações gerais quanto ao rebanho, às atividades desenvolvidas, além da produção de leite, dos dados de produção e qualidade do leite, da área destinada a cada atividade, das pastagens (tipo e manejo), da disponibilidade de equipamentos e infraestrutura, da mão de obra utilizada e das informações acerca da família do produtor. Nessa ocasião era assinado um termo de anuência do produtor em participar das atividades do projeto e, em sequência, era aplicada a ferramenta de diagnóstico de boas práticas.

Todos os técnicos receberam treinamento teórico e prático antes de aplicarem a ferramenta Protambo. O treinamento teórico consistiu na familiarização com o método, por meio de leitura e de discussão dos indicadores utilizados na ferramenta, para nivelar conceitos e compreensão da aplicação da escala de avaliação. O treinamento em campo foi a aplicação da ferramenta em, pelo menos, duas UPL acompanhadas pelo técnico avaliador, sob supervisão do orientador (avaliador de referência). Durante essa aplicação, as situações encontradas eram discutidas entre ambos.

Para utilização adequada da ferramenta, o técnico deve acompanhar a rotina da propriedade, de maneira a incluir a observação de, pelo menos, uma ordenha do começo ao fim e percorrer a propriedade em toda a sua extensão, da porteira de entrada às áreas de preservação permanente. Acima de tudo, o técnico deve estar preparado para observar o que é feito, onde, quando, como e quem executa as tarefas, bem como observar interações entre pessoas, pessoas e animais, entre animais, percorrer todos os locais, todas as instalações e acessar os registros de rotinas, quando existirem. Os técnicos foram orientados a perguntar somente o estritamente necessário, pois a ferramenta é um roteiro de indicadores e não um questionário.

A ferramenta Protambo de diagnóstico de BPA na pecuária de leite consiste em um roteiro de indicadores das práticas adotadas para as seis áreas-chave de boas práticas relacionadas no guia FAO e IDF (Guia, 2013): sanidade animal; higiene na ordenha; nutrição (alimentos e água); bem-estar animal; meio ambiente; e gestão socioeconômica. Contempla aspectos objetivos e demonstráveis, que permitem avaliar a adoção de boas práticas em sistemas de produção leiteira. Os indicadores são informações/ações ou situações que podem ser identificadas a partir de 
entrevista, inspeção direta e análises laboratoriais de apoio, como qualidade da água, dos alimentos, do leite, do solo, testes diagnósticos sanitários, entre outros.

Os indicadores adotados para cada uma das seis áreas-chave da ferramenta são agrupados por similaridade e complementaridade, de modo a abranger e diagnosticar a situação das diferentes práticas/processos essenciais ao escopo de cada área-chave. Assim, a área-chave sanidade animal contempla oito grupos de indicadores das seguintes práticas (1-vacinações, 2-diagnóstico de tuberculose e brucelose, 3-controle de endo e ectoparasitas, 4- uso e armazenamento de vacinas e medicamentos, 5-trânsito e introdução de animais, 6-acompanhamento sanitário, 7controle de pragas e vetores de doenças, 8histórico reprodutivo). As demais áreas-chave contemplam cinco grupos cada, quais sejam: higiene na ordenha (1-procedimentos de ordenha, 2-higiene do ordenhador, 3-higiene do local de ordenha, 4-higiene dos utensílios e equipamentos, 5-higiene do leite após ordenha); nutrição (1-avaliação da condição corporal e do desenvolvimento dos animais, 2-oferta e controle de qualidade dos alimentos, 3-produção de forragem, 4-oferta e qualidade da água, 5segurança dos alimentos oferecidos e da produção de forragem); bem-estar animal (1condição geral do rebanho, 2-interações homemanimal, 3-instalações e conforto animal, 4equipamentos/procedimentos de ordenha $\mathrm{e}$ conforto animal, 5-manejo sanitário e bem-estar animal); meio ambiente (1-tratamento de efluentes, 2-proteção de mananciais e vegetação de áreas de preservação permanente, 3-manejo do solo, 4-uso de defensivos e descarte de embalagens e resíduos, 5-ordem e asseio geral); gestão socioeconômica (1- controle zootécnico, 2-práticas de gestão financeira e contábil, 3práticas trabalhistas e gestão de mão de obra, 4higiene e segurança do trabalho, 5-avaliação de risco de investimentos). No total são avaliados 33 grupos de indicadores em cada aplicação da ferramenta a uma dada UPL.

A escala de avaliação estabelecida considera dois níveis acima e dois abaixo do ponto central, que indica o padrão mínimo de conformidade dos indicadores, de maneira a atender a necessidade de se identificar o nível de adoção das BPA, com detalhamento suficiente para permitir a estratificação nos níveis descritos, porém fugindo à excessiva complexidade que uma escala com mais níveis poderia suscitar. Cada grupo de indicadores recebe uma avaliação na escala a seguir:

abaixo do esperado (-2); abaixo do esperado (-1); dentro do esperado (0); acima do esperado (1); e acima do esperado (2).

No momento da aplicação da ferramenta, o foco deve ser no uso da escala numérica, cuja variação obedece aos critérios descritivos dos grupos de indicadores de cada área-chave. Portanto, evita-se o uso de advérbios de intensidade para categorizar o nível identificado das práticas, definido pela pontuação da escala e descrito apenas como acima, abaixo, ou dentro do esperado. Na etapa seguinte, os resultados dos grupos de indicadores em cada área-chave definem o nível de adoção de boas práticas, conforme a seguir:

Padrão de excelência: adoção de práticas de forma a obter resultados máximos possíveis em período de tempo que demonstre a consistência dessa adoção. O tempo necessário para demonstrar essa consistência pode variar conforme o indicador em questão.

Padrão de referência: adoção de práticas de forma a obter resultados acima dos níveis esperados em período de tempo que demonstre a consistência dessa adoção. O tempo necessário para demonstrar essa consistência pode variar conforme o indicador em questão.

Padrão de conformidade: as BPA são constatadas e seus efeitos são observáveis no contexto de sua aplicação ou pela ausência de dano a ser evitado.

Práticas insuficientes: situação que caracteriza a adoção de práticas inadequadas, ineficazes, inconsistentes, mal executadas, com resultados abaixo do esperado, ou inexistentes.

Práticas precárias: situação que caracteriza a inexistência de determinadas práticas ou práticas inadequadas, ineficazes, inconsistentes ou mal executadas, com resultados abaixo do esperado, ou inexistentes, em indicadores que ameacem diretamente a segurança do alimento. 
Para avaliação das áreas, alguns indicadores são priorizados segundo sua interferência sobre o resultado econômico da atividade e sobre a segurança do alimento, com prioridade absoluta para o último aspecto. Abaixo segue como exemplo a classificação dos resultados utilizada para avaliar a área-chave de sanidade animal.

Avaliação da área-chave sanidade animal:

Práticas precárias: presença de três ou mais itens com classificação $(-1)$ no total e/ou avaliação (-2) nos grupos 1, 2 e 6 - necessidade de ação imediata.

Práticas insuficientes: até quatro (0) e não mais que uma avaliação (-2) nos grupos $3,4,5,7$ e 8 necessidade de ação urgente.

Padrão de conformidade: ausência de grupos (2) e (-1).

Padrão de referência: pelo menos quatro grupos (1) e nenhum abaixo de (0).

Padrão de excelência: acima de quatro grupos (1) e pelo menos um grupo (2).

Os grupos de indicadores 1, 2 e 6 são prioritários na avaliação da área-chave sanidade animal. A avaliação (-2) em qualquer um deles demonstra a precariedade das práticas adotadas na fazenda em relação aos requisitos do Guia FAO-IDF (Guia, 2013). Da mesma forma, três ou mais avaliações (-1) nos grupos $3,4,5,7$ e 8 configuram situação de alta vulnerabilidade sanitária do rebanho, representando risco para a segurança do alimento, das pessoas e para o resultado econômico da atividade. O mesmo critério de priorização é aplicado às demais áreas.

Assim, o diagnóstico configura-se com base nos resultados encontrados para os grupos de indicadores e a subsequente identificação das áreas-chave críticas. Essa identificação permite o estabelecimento de planos de ajuste para a correção de não conformidades, de acordo com o respectivo impacto potencial sobre a segurança do alimento, a eficiência do sistema de produção e os recursos naturais. Quanto ao último aspecto, a adoção de boas práticas agropecuárias na áreachave meio ambiente pode ser imediata ou não, mas nem sempre dessa celeridade na implementação de medidas se segue a remissão ou a mitigação imediata dos danos. Cumpre destacar, também, que os planos de ação são específicos para cada UPL. Por meio do acompanhamento da realização dos planos de ação e da reaplicação da ferramenta Protambo, pode ser medida a evolução de cada UPL segundo a variação dos próprios indicadores em momentos distintos, como também é possível se analisar a evolução de grupos de propriedades submetidas ao mesmo método.

As unidades de observação da pesquisa foram as fazendas de produção de leite incluídas no projeto Protambo, totalizando 62 UPL estudadas. As análises estatísticas foram feitas segundo critérios de validação conforme a norma ISO/IEC 17025, para certificação da competência de laboratórios de ensaio e calibração (General, 2005). Desse quadro amostral, 14 (catorze) UPL foram submetidas ao diagnóstico e à aplicação de um plano de ação (grupo tratamento) e 12 (doze) unidades foram consideradas como grupo controle. Nas propriedades controle, foi estabelecido o diagnóstico, mas sem ações sistemáticas de ajuste das não conformidades, configurando-se tamanho amostral de aproximadamente $20 \%$ do total de propriedades estudadas, o que possibilita análises estatísticas com resultados confiáveis (Cochran, 1977). O grupo controle incluiu 12 UPL sorteadas entre aquelas que, tendo sido incorporadas inicialmente ao projeto, não receberam acompanhamento por parte dos técnicos e não tiveram plano de ajuste de inconformidades executado. $\mathrm{O}$ grupo tratamento incluiu 14 UPL cujo acompanhamento e execução dos planos de ação foi realizado conforme o acordo inicial estabelecido entre técnicos, produtores e equipe do projeto (periodicidade mínima de visitas, plano de ação pactuado com o produtor e execução das ações) no momento da seleção da propriedade.

Para avaliação do grupo controle, foi verificado se houve diferença entre a avaliação inicial dos 33 grupos de indicadores em cada UPL quando da inclusão na pesquisa (2014) e ao final (2017). O grupo tratamento foi avaliado da mesma forma, porém foram implementadas ações de ajuste de não conformidades conforme os planos de ação elaborados.

A validação da ferramenta Protambo foi realizada comparando-se as diferenças entre os resultados obtidos por diferentes avaliadores em um determinado momento, na mesma propriedade, para cada grupo de indicadores. $\mathrm{O}$ resultado de cada um dos 33 (trinta e três) grupos de indicadores do avaliador técnico foi 
comparado aos mesmos resultados dos 33 grupos de indicadores do avaliador referência. Foram realizadas 17 observações da aplicação da ferramenta por oito diferentes técnicos e pelo avaliador referência, simultaneamente, ou em intervalos de tempo inferiores ao mínimo considerado para que a interferência de fatores externos, tais como estação do ano, oferta de alimentos, e demais fatores conjunturais pudesse gerar diferenças além das possíveis divergências entre avaliadores.

A análise estatística foi realizada utilizando-se o escore $Z$, comumente empregado na avaliação de desempenho de laboratórios em ensaios analíticos ou bioanalíticos no âmbito de programas interlaboratoriais de proficiência (Procedimento, 2000). O escore $\mathrm{Z}$ permite que se saiba o quanto um determinado dado se afasta da média de uma amostra em número de desviospadrão. A aplicação do escore $\mathrm{Z}$ possibilita, portanto, a validação da ferramenta Protambo pela verificação da coerência entre os resultados obtidos quando da aplicação por diferentes avaliadores e a análise da variação dos indicadores entre sucessivas aplicações da ferramenta.

Para ordem de cálculo do escore Z, foi utilizada a fórmula abaixo :

$$
\mathrm{Z}=(\mathrm{X}-\mu) / \sigma \text {, }
$$

em que: $X$ é a nota atribuída ao grupo de indicadores, $\mu$ foi estimado pela média amostral das notas dos 33 grupos de indicadores e $\sigma$ é o desvio-padrão estimado na amostra. $\mathrm{O}$ parâmetro estabelecido nesse caso é similar à exatidão propagada pela ISO/IEC 17025 para certificação da competência de laboratórios de ensaio e calibração.

Para validação da ferramenta, foram considerados inválidos resultados com mínimo de quatro escores $\mathrm{Z} \mathrm{C}(-2,2)$, ou seja, $|\mathbf{Z}|>\mathbf{2}$, assim, máximo de não conformidades de quatro escores Z (Gonçalves e Alves, 2007). Os resultados válidos (rejeitada a hipótese de nulidade) foram aqueles em que os valores atribuídos pelo avaliador referência e pelo técnico diferem, segundo o parâmetro acima, em quatro ou mais grupos de indicadores $(B \geq 4)$, do total de 33 (trinta e três) analisados.

A evolução dos indicadores nos grupos controle e tratamento foi analisada segundo distribuição binomial para o número de avaliações crescentes (B) e respectivas probabilidades $(P(B))$, em que $B$ é o número de vezes em que houve crescimento entre a avaliação inicial e a final $(\mathrm{B} \geq 4)$. Finalmente, foram comparadas as variações entre os grupos controle e tratamento. Assim, o principal modelo estatístico empregado foi a distribuição binomial de probabilidades para o número de variações positivas entre início e fim das avaliações nos grupos presentemente denominados controle e tratamento (NIST/SEMATECH, 2017; Kendall et al., 1994). Foi também empregada a aproximação normal para o teste t-Student para comparação entre os grupos controle e tratamento (Conover, 1999). Essa aproximação deriva da distribuição normal aproximada de $\mathrm{Z}[\mathrm{Z}=(\mathrm{B}-\mathrm{E}(\mathrm{B})) / \sigma(\mathrm{B})]$, em que $\mathrm{E}(\mathrm{B})$ é o valor esperado de $\mathrm{B}$, e $\sigma(\mathrm{B})$ o desviopadrão. Os níveis de significância usados na análise variaram entre $0,05 \quad(95 \%$ de confiabilidade) para testes de evolução nas avaliações dos grupos de indicadores. Não houve comparação entre as UPL (unidades de observação), mas sim mensuração da variação dos 33 grupos de indicadores em cada unidade de observação.

\section{RESULTADOS E DISCUSSÃO}

Os resultados da aplicação da ferramenta Protambo (Tab. 1) mostram baixo nível de adoção de boas práticas em todas as áreas-chave diante das recomendações contidas no Guia FAO-IDF. Os resultados obtidos, embora não representem o universo dos produtores do Rio Grande do Sul ou das regiões de abrangência do projeto, são indicativos de riscos preocupantes à segurança do leite no contexto analisado e convergem para os dados de diversos outros levantamentos sobre a qualidade do leite e BPA no Brasil (Brito, 2004; Vallin et al., 2009; Mororó, 2011). A segurança sanitária da cadeia produtiva, nas condições encontradas nesta pesquisa, depende fundamentalmente da pasteurização e dos demais tratamentos térmicos em suas diferentes modalidades. A maior parte do leite no universo de propriedades examinado neste trabalho é produzido sob condições e práticas abaixo dos requisitos mínimos (Guia, 2013) para consumo "cru" ou de derivados não pasteurizados. Os resultados encontrados nesta pesquisa, especialmente para as áreas chave de sanidade animal e manejo de ordenha vão ao encontro das constatação de Vallin et al. (2009), 
no Brasil, e de Van Soest et al. (2016), na Holanda, segundo os quais o manejo inadequado de ordenha dos animais, a ocorrência de mastites, a contaminação do leite por deficiência de higienização de equipamentos e de refrigeração estão entre os pontos determinantes das elevadas contagens de células somáticas e de bactérias e de prejuízos extensivos a toda a cadeia de lácteos.

Tabela 1. Nível de adoção de BPA segundo as áreas-chave de boas práticas agropecuárias do Guia FAOIDF nas 62 UPL participantes do projeto Protambo, dados referentes ao ano de 2015

\begin{tabular}{lcccccc}
\hline $\begin{array}{c}\text { Nível de adoção } \\
\text { das BPA }\end{array}$ & $\begin{array}{c}\text { Sanidade } \\
\text { animal }\end{array}$ & $\begin{array}{c}\text { Higiene } \\
\text { ordenha }\end{array}$ & Nutrição & $\begin{array}{c}\text { Bem- } \\
\text { estar }\end{array}$ & $\begin{array}{c}\text { Meio } \\
\text { ambiente }\end{array}$ & Gestão \\
\hline Excelência & 0 & 0 & 0 & 0 & 0 & 0 \\
Referência & 1 & 2 & 0 & 1 & 0 & 0 \\
Conforme & 1 & 3 & 1 & 2 & 2 & 3 \\
Insuficiente & 6 & 23 & 9 & 20 & 9 & 6 \\
Precária & 54 & 34 & 52 & 39 & 51 & 53 \\
Total & 62 & 62 & 62 & 62 & 62 & 62 \\
\hline
\end{tabular}

É consenso técnico e determinação legal que todo o leite consumido como bebida ou destinado à produção de derivados no Brasil deva ser pasteurizado (a não ser a recente exceção feita a alguns queijos artesanais, em alguns estados do país). A despeito desse "anteparo de segurança" representado pela pasteurização e por outros tratamentos térmicos, a vida de prateleira, os atributos sensoriais, nutricionais e o potencial de agregação de valor via produção de derivados ficam seriamente prejudicados pela qualidade comprometida do leite captado. Esse conjunto de problemas limita a competitividade do setor de lácteos no mercado interno, impede o Brasil de exportar e remete à necessidade de adoção das boas práticas agropecuárias nas fazendas. Porém, as BPA, por si mesmas, não resolvem se não forem amparadas por referenciais que apontem o que, quanto, quando, como, onde, por que e quem deve ser responsável em cada etapa por sua adoção nas fazendas.

Abordagens participativas com maior ou menor aporte de recursos públicos e de compartilhamento de responsabilidades com os produtores, como a Rede Leite, no noroeste do RS (Silva, 2010), o Projeto Vitória, do Instituto Emater-PR, a Rede de Propriedades de Referência para Agricultura Familiar Iapar/Instituto Emater-PR, o Projeto Rede de Transferência Tecnológica em Sistemas de Produção de Leite em Pasto da Coopeler-PR (Soares Júnior, 2006; Coelho Junior et al., 2012; Llanillo et al., 2012), entre outros, têm sido adotadas com sucesso variável. Todas essas experiências envolvem o estímulo à adoção de boas práticas, mas nenhuma delas trabalha com o enfoque sistêmico baseado nas recomendações do Guia FAO/IDF, por meio do uso de uma ferramenta nos moldes da ferramenta Protambo. Experiência internacional comparável, mas que adota outros recursos, é o programa australiano "Countdown Downunder", baseado no conceito de "Construção de Capacidade" ("CapacityBuilding"). Trata-se do aumento das habilidades e dos recursos dos indivíduos, das organizações e das comunidades para administrar a mudança, a partir das seis etapas do ciclo de planejamento de ação (PDCA): identificar necessidades, estabelecer objetivos, planejar a ação, executar, revisar o processo, aprender e replanejar. São ministrados cursos breves para produtores e são utilizados recursos, como cartilhas, planos de ação, relatórios focais sobre mastite e prêmios de qualidade do leite. O programa foi creditado como sendo o elemento fundamental para a melhoria da qualidade do leite australiano em curto espaço de tempo, particularmente no tocante aos desafiadores indicadores de mastite (More, 2009).

Os resultados mostrados na Tab. 1 evidenciam também que, além das práticas deficientes nas áreas-chave sanidade animal e higiene da ordenha, com impactos diretos e imediatos na segurança e qualidade do leite, as demais áreaschave também não contam com a aplicação das BPA. Essas áreas representam oportunidade de grandes ganhos de eficiência do sistema de produção como um todo, além do impacto na qualidade e segurança do alimento. As BPA nas áreas-chave de nutrição, bem-estar animal e gestão socioeconômica têm alto impacto 
potencial sobre o resultado financeiro do sistema de produção e podem ser ponto de partida para mudanças de percepção dos produtores acerca da importância das BPA.

Prover técnicos e produtores de uma ferramenta que permita a identificação sistemática e com indicadores sensíveis, demonstráveis, pode ser um importante passo inicial para sensibilização. Para técnicos em formação ou inexperientes, a ferramenta pode ser utilizada em programas de capacitação e como guia para compreensão sistêmica das UPL. Para os profissionais experientes, pode facilitar a elaboração e pactuação com os produtores dos planos de ação para adoção das BPA, bem como o monitoramento de sua evolução. Os produtores, por seu turno, podem se beneficiar de um diagnóstico do que acontece nas suas fazendas e da elaboração conjunta com os técnicos de planos de ação adequados à sua realidade.

A Tab. 2 apresenta os resultados da aplicação da ferramenta por distintos técnicos avaliadores, comparados ao avaliador de referência. B registra o número de vezes em que houve discrepância, medida em escores $Z$, entre os valores atribuídos pelos técnicos avaliadores e o avaliador de referência, a cada um dos 33 grupos de indicadores adotados na ferramenta, em cada observação. Verifica-se que, em 561 resultados (17observações $\times 33$ grupos de indicadores,= 561), apenas 24 resultados divergiram significativamente. Somente quatro entre as 17 observações dos técnicos (primeira, quinta, nona e $16^{\mathrm{a}}$ observações) se mostraram significativamente $(\mathrm{P}>0,05)$ não conformes quando comparadas com o avaliador referência, $\mathrm{p}(\mathrm{B} \geq 4)=0,977$, satisfazendo o parâmetro de exatidão da ISO/IEC 17025. Assim, os resultados validam a aplicação da ferramenta por distintos avaliadores.

Os resultados da evolução dos indicadores no grupo controle (Tab. 3) denotam tendência de diferença significativa nos indicadores entre a avaliação final e a inicial das UPL, com o emprego da ferramenta Protambo, conforme indicado por $\mathrm{B}$ e $\mathrm{P}(\mathrm{B})$. Isso demonstra a sensibilidade da ferramenta às alterações nos indicadores ao longo do tempo, de acordo com o nível de adoção de práticas nas UPL, ou seja, uma forma de tendência que valida a ferramenta. Detectou-se, portanto, forma de métrica de sensibilidade, bem como similaridade à linearidade, outro parâmetro exigido pela ISO/IEC 17025 em validação de métodos analíticos de medição. As UPL nesse grupo receberam a aplicação da ferramenta de diagnóstico, mas sem aplicação do plano de ação para ajuste das não conformidades encontradas.

Tabela 2. Resultados da aplicação da ferramenta por distintos técnicos avaliadores

\begin{tabular}{llll}
\hline Observações & NumPro & Técnico & B \\
\hline 1 & 23 & A & $5^{*}$ \\
2 & 24 & A & 2 \\
3 & 25 & A & 3 \\
4 & 26 & A & 1 \\
5 & 23 & B & $6^{*}$ \\
6 & 24 & B & 1 \\
7 & 25 & B & 0 \\
8 & 26 & B & 1 \\
9 & 19 & C & $8^{*}$ \\
10 & 12 & D & 3 \\
11 & 20 & D & 0 \\
12 & 21 & D & 1 \\
13 & 22 & D & 1 \\
14 & 19 & E & 1 \\
15 & 27 & F & 1 \\
16 & 19 & G & $5^{*}$ \\
17 & 19 & H & 3 \\
\hline
\end{tabular}

B: registra o número de vezes em que, nos 33 grupos de indicadores, houve discrepância entre avaliadores e avaliador referência.

*: $\mathrm{B} \geq 4$, divergência significativa $(\mathrm{P} \leq 0,05)$. NumPro: número da propriedade. Local: localização.

Tabela 3. Evolução dos indicadores no grupo controle

\begin{tabular}{cccc}
\hline Observação & NumPro & Local & B \\
\hline 1 & 1 & $\mathrm{~A}$ & $4^{*}$ \\
2 & 2 & $\mathrm{~A}$ & 8 \\
3 & 3 & $\mathrm{~A}$ & 9 \\
4 & 4 & $\mathrm{~A}$ & $5^{*}$ \\
5 & 5 & $\mathrm{~A}$ & $7^{*}$ \\
6 & 6 & $\mathrm{~B}$ & $4^{*}$ \\
7 & 7 & $\mathrm{~B}$ & $0^{*}$ \\
8 & 8 & $\mathrm{~B}$ & $1^{*}$ \\
9 & 9 & $\mathrm{~B}$ & $5^{*}$ \\
10 & 19 & $\mathrm{C}$ & $1^{*}$ \\
11 & 11 & $\mathrm{C}$ & $3^{*}$ \\
12 & 12 & $\mathrm{D}$ & $6^{*}$ \\
\hline
\end{tabular}

B: número de indicadores em que houve diferença entre a avaliação inicial e a final $(B \geq 4)$.*: $\mathrm{P}(\mathrm{B})<0,001$ NumPro: número da propriedade. Local: localização. 
No entanto, a dependência intrínseca do acompanhamento das UPL pelos técnicos pode ser verificada como uma das limitações da metodologia adotada neste estudo. As propriedades do grupo controle foram selecionadas entre aquelas inicialmente incluídas no projeto Protambo, mas não foram acompanhadas como previsto. A interrupção foi motivada por diversos fatores, como a impossibilidade de a instituição parceira manter compromissos com os técnicos contratados, a falta de recursos para manutenção de veículos e combustível para deslocamentos, desligamento voluntário de técnicos, desistência de produtores, entre outros. Esses fatores limitaram o espaço amostral inicial de 62 (sessenta e duas) propriedades, de modo que 12 (doze) puderam ser consideradas controle e outras 14 (catorze) como tratamento. As restantes 38 (trinta e oito) UPL tiveram seus acompanhamentos interrompidos ou execução parcial do plano de ação, limitando sua inclusão nesta pesquisa, embora tenham sido mantidas como participantes do projeto. Esse cenário aponta para a fragilidade das estruturas de assistência técnica e de extensão no ambiente estudado, no que diz respeito à consistência do acompanhamento de UPL. Para que se avance na solução dos problemas da cadeia de produção de leite e derivados, apontados desde a introdução desse documento, é necessária a criação de programas de assistência técnica que contemplem frequência de acompanhamento, sequência de ações e duração em período suficiente para que seus objetivos sejam atingidos.

A Tab. 4 apresenta os dados referentes à evolução dos indicadores durante o acompanhamento no grupo tratamento. As UPL nesse grupo receberam a aplicação da ferramenta para o diagnóstico do nível das práticas adotadas e a subsequente aplicação do plano de ação para solução das não conformidades encontradas. Os resultados evidenciam a evolução positiva significativa $(\mathrm{P}<0,05)$ dos indicadores entre a avaliação inicial e a final. $O$ espaço de variação encontrado demonstrou desenvolvimento de métrica, precisão e consistência (coerência) de medição na ferramenta Protambo. Apenas uma UPL não mostrou melhora significativa no nível de BPA (uma em 14), demonstrando excelente desempenho para um início de treinamento e programa.
Tabela 4. Evolução dos indicadores no grupo tratamento

\begin{tabular}{lllc}
\hline Observações & NumPro & Local & B \\
\hline 1 & 20 & $\mathrm{D}$ & $8^{*}$ \\
2 & 21 & $\mathrm{D}$ & $7^{*}$ \\
3 & 22 & $\mathrm{D}$ & $17^{*}$ \\
4 & 27 & $\mathrm{E}$ & $4^{*}$ \\
5 & 28 & $\mathrm{E}$ & $13^{*}$ \\
6 & 30 & $\mathrm{E}$ & $6^{*}$ \\
7 & 31 & $\mathrm{E}$ & $12^{*}$ \\
8 & 32 & $\mathrm{E}$ & $8^{*}$ \\
9 & 34 & $\mathrm{~F}$ & $7^{*}$ \\
10 & 35 & $\mathrm{~F}$ & $4^{*}$ \\
11 & 36 & $\mathrm{~F}$ & $7^{*}$ \\
12 & 37 & $\mathrm{~F}$ & 2 \\
13 & 38 & $\mathrm{~F}$ & $4^{*}$ \\
14 & 39 & $\mathrm{~F}$ & $4^{*}$ \\
\hline
\end{tabular}

$\mathrm{B}$ : número de indicadores em que houve crescimento entre a avaliação inicial e a final *:crescimento significativo ( $\mathrm{P} \leq 0,05$ para $\mathrm{B} \geq 4$ ), entre a avaliação inicial e a final. NumPro: número da propriedade. Local: localização.

O valor da estatística t-Student obtido para a comparação de médias de não conformidades entre os grupos controle e tratamento (Conover, 1999) obtidas resultou em $\mathrm{t}=-2,133 ; \mathrm{P}=0,021$. Esse resultado evidencia que houve diferença significativa entre os indicadores de adoção de BPA entre os grupos, em um nível menor que $5 \%$ de probabilidade. Os resultados obtidos indicam que a ferramenta de diagnóstico Protambo foi validada segundo padrões internacionais e apresenta três parâmetros essenciais para os fins a que se destina: sensibilidade às variações (métrica); precisão (exatidão dentro de limites aceitáveis para medições na área); e consistência (coerência na medição)

\section{CONCLUSÕES}

A ferramenta Protambo de diagnóstico da adoção e conformidade das práticas adotadas em fazendas de produção de leite apresentou os requisitos segundo padrões internacionalmente reconhecidos, para ser utilizada em programas de fomento às BPA na produção de leite. A ferramenta Protambo possibilitou a avaliação sistemática e a abordagem sistêmica para conhecimento da realidade quanto à observância das boas práticas agropecuárias nas fazendas nas quais foi aplicada. A utilização de indicadores detalhados e demonstráveis permitiu 
fundamentar a elaboração e o comprometimento dos produtores com planos para ajuste de não conformidades.

\section{REFERÊNCIAS}

BRITO, J.R.F. Adoção de boas práticas agropecuárias em propriedades leiteiras da Região Sudeste do Brasil como um passo para a produção de leite seguro. Acta Sci. Vet., v.32, p.125-131, 2004.

COCHRAN, W.G. Sampling techniques. 3.ed. New York: Wiley, 1977. 442p.

COELHO JUNIOR, L.M.; MIRANDA, M.; FUENTES LLANILLO, R.; SOARES JUNIOR, D. Análise comparativa de sistemas de baixa e média intensidade de produção de leite, em bases ecológicas, no Oeste do Paraná. In: CONGRESSO DA SOCIEDADE BRASILEIRA DE ECONOMIA, ADMINISTRAÇÃO E SOCIOLOGIA RURAL, 50., 2012, Vitória. Anais...Vitória: [s.n.], 2012.

CONOVER, W.J. Practical nonparametric statistics. New York: Wiley, 1999. 592p.

COSTA, H.B.A.; DANTAS, R.M.; ALVARENGA, M.B. et al. Programs for best dairy management practice in Brazil and their applications. J. Cleaner Prod. v.176, p.1026-1033, 2018.

DUFOUR, S. Manageable risk factors associated with the lactational incidence, elimination, and prevalence of Staphylococcus aureus intramammary infections in dairy cows. J. Dairy Sci., v.95 p.1283-1300, 2012.

GENERAL requirement for the competence of testing and calibration laboratories. ISO/IEC 17025. Genéve: ISO, 2005

GONÇALVES E.B.; ALVES A.P.G. Análise sequencial na evidência de competência de laboratórios de análises por ensaios de proficiência. Braz. J. Food Technol., v.10, p.194-197, 2007.

GUIA de boas práticas na pecuária de leite. Produção e saúde animal, diretrizes. Roma: FAO/IDF, 2013.

KENDALL, M.G et al. Advanced theory of statistics. New York: Wiley, 1994. v.2, part.1, 912 p.

LLANILLO, R.F.; ALMEIDA, E.L.D.; DIMAS S.; MIRANDA, M.; CARNEIRO, S.L. Redes de inovação e redes de difusão: ampliação do enfoque sistêmico no estado do Paraná. In: CONGRESSO DA SOCIEDADE BRASILEIRA DE SISTEMAS DE PRODUÇÃO, 9., 2012, Luziânia. Anais... Luziânia: SBSP, 2012.
MORE, S. Global trends in milk quality: implications for the Irish dairy industry. Ir. Vet. J., v.62, Suppl.4, p.S5-14, 2009.

MORORÓ, A.M. Qualidade do leite caprino antes da aplicação de boas práticas agropecuárias: estudo de casos. CONGRESSO BRASILEIRO DE ZOOTECNIA, 21., 2011, Maceio. Anais... Maceio: Universidade Federal de Alagoas, 2011. (Resumo Expandido)

NIST/SEMATECH. Engineering Statistics Handbook. Disponível em:

<http://www.itl.nist.gov/div898/handbook/>. Acesso em: 9 maio 2017.

PROCEDIMENTO sobre a participação dos laboratórios de ensaios na atividade de ensaio de proficiência. NIT - DICLA 026. Rio de Janeiro: INMETRO, 2000. 6p.

SCALCO, A.R.; SOUZA, R.C. Qualidade na cadeia de produção de leite: diagnóstico e proposição de melhorias. Org. Rural Agroind., v.8, p.368-377, 2006.

SILVA, G.M. Rede leite: programa em rede de pesquisa-desenvolvimento em sistemas de produção com pecuária de leite no noroeste do Rio Grande do Sul. Bagé: Embrapa Pecuária Sul, 2010. 22p. (Documentos 100).

SOARES JÚNIOR, D.A. Organização de redes de unidades produtivas como instrumento de apoio ao desenvolvimento territorial rural. 2006. 142f. Dissertação (Mestrado em Administração) Universidade Estadual de Maringá, PR.

VALLIN, V.M.; BELOTI, V.; BATTAGLINI, A.P.P. et al. Melhoria da qualidade do leite a partir da implantação de boas práticas de higiene na ordenha em 19 municípios da região central do Paraná. Semin. Ciênc. Agrar., v.30, p.181-188, 2009.

VAN SOEST, F.J. et al. Failure and preventive costs of mastitis on Dutch dairy farms. J. Dairy Sci., v.99, p.8365-8374, 2016.

YOUNG, I.; RAJIĆ A.; HENDRICK, S. et al. Attitudes towards the Canadian quality milk program and use of good production practices among Canadian dairy producers. Prev. Vet. Med., v.94, p.43-53, 2010. 